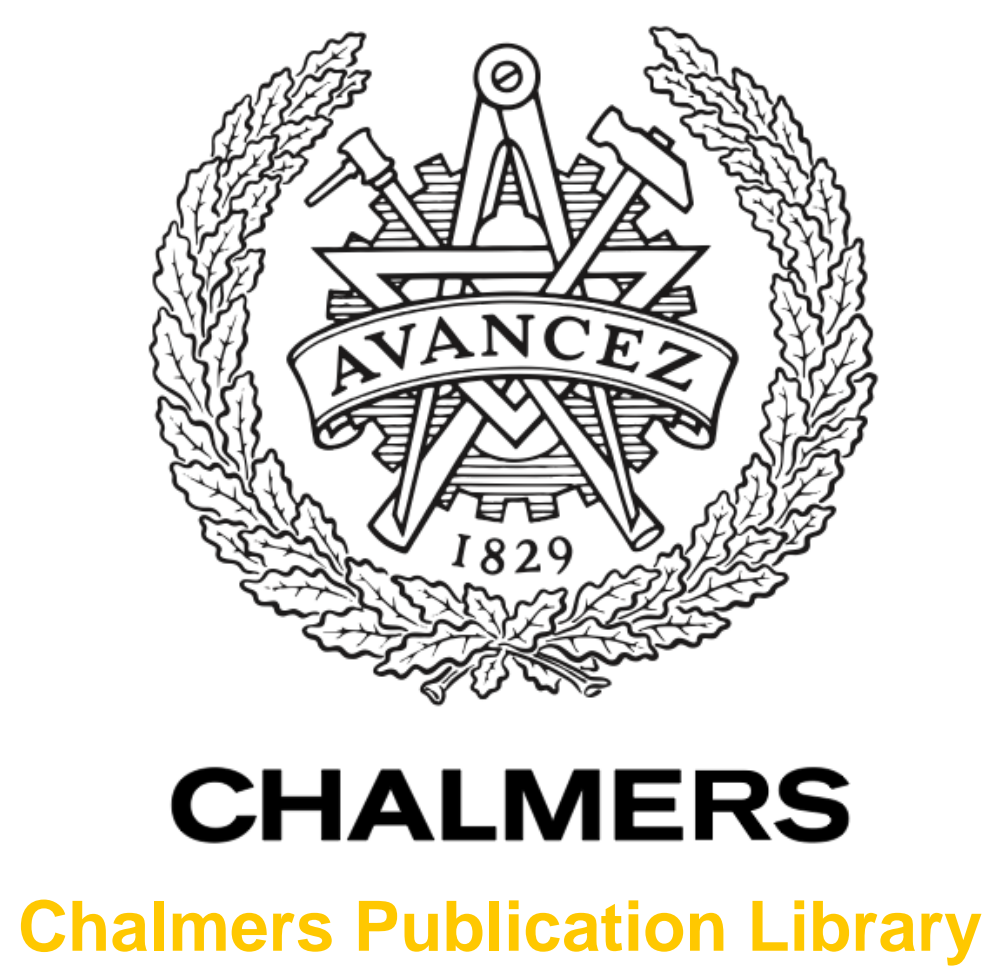

\title{
Location-Aware Formation Control in Swarm Navigation
}

This document has been downloaded from Chalmers Publication Library (CPL). It is the author's version of a work that was accepted for publication in:

\section{IEEE Globecom Workshops}

Citation for the published paper:

Zhang, S. ; Fröhle, M. ; Wymeersch, H. et al. (2015) "Location-Aware Formation Control in Swarm Navigation". IEEE Globecom Workshops

Downloaded from: http://publications.lib.chalmers.se/publication/228413

Notice: Changes introduced as a result of publishing processes such as copy-editing and formatting may not be reflected in this document. For a definitive version of this work, please refer to the published source. Please note that access to the published version might require a subscription. 


\title{
Location-Aware Formation Control in Swarm Navigation
}

\author{
Siwei Zhang ${ }^{1}$, Markus Fröhle ${ }^{2}$, Henk Wymeersch ${ }^{2}$, Armin Dammann ${ }^{1}$ and Ronald Raulefs ${ }^{1}$ \\ ${ }^{1}$ Institute of Communications and Navigation, German Aerospace Center (DLR), Germany \\ Email: firstname.lastname@dlr.de \\ ${ }^{2}$ Department of Signals and Systems, Chalmers University of Technology, Sweden \\ Email: \{frohle, henkw\}@chalmers.se
}

\begin{abstract}
Goal-seeking and information-seeking are canonical problems in mobile agent swarms. We study the problem of collaborative goal-approaching under uncertain agent position information. We propose a framework that establishes locationaware formations, resulting in a controller that accounts for agent position uncertainty with a realistic ranging model. Simulation results confirm that, as the outcome of the controller, the swarm moves towards its goal, while emerging formations conducive to high-quality localization.
\end{abstract}

\section{INTRODUCTION}

Swarms of mobile agents, such as robots and drones, can be utilized to accomplish a wide variety of tasks, including goalseeking and forming formation [1], based on collaboration. For instance, moving a swarm towards a goal position, while maintaining precise position information of each agent relies on explicit coordination, both to improve the global position estimate of each agent, and to reduce the risk of collisions with known obstacles. Accomplishing these tasks relies on accurate and timely position information, which is often overlooked in the technical literature. Existing approaches, such as [2][6], assume perfect position information, which may not be available in practical scenarios.

The objectives of goal-approaching and precise localization generally rely on some form of range-based distance estimate. Distance estimation can be performed with anchor nodes, but also collaboratively by the agents inside the swarm, reducing the need of adding many anchors on the path towards the goal. Therefore, the limited coverage of the anchor signals is compensated by integrating cooperative range estimates between close-by agents to determine the position of all mobile agents. Cooperative positioning with radio nodes based on belief propagation algorithms was presented in [7] and extended in [8] to account for accessing the radio channel by a time-division multiple access protocol using an orthogonalfrequency division multiplexing (OFDM) signal. Cramér-Rao bound (CRB) introduced in [9] provides the fundamental limits of the cooperative positioning accuracy.

In [10], the authors indicated that the localization errors must be considered for formation control, e.g., flocking. Complementary, in [11], [12] the authors have the explicit aim to control agents to improve or maintain the positioning quality through information-seeking. The information quality is closely linked to the ranging model, which can be characterized through bounding techniques, such as the CRB and the Ziv-Zakai bound (ZZB) [13], or with a knowledge of ranging variance.

In this work, we combine goal approaching and informationseeking through an alternating optimization formulation with a realistic distance-dependent ranging model. The goal approaching objective forces agents to move to their goal, while the information seeking objective forces agents to form configurations that are conducive for high-precision localization. We provide a description of the two objective functions as well as a gradient descent controller based on positioning CRB. Simulation results confirm that a realistic ranging model is essential for generating an effective swarm controller. The swarm moves in a way such that it reaches the goals, while cooperatively maintaining good localization performance.

\section{System AND PRoblem Formulation}

\section{A. Dynamic System Formulation}

We consider a network comprising $B$ base stations (BSs) and $M$ agents (AGs). The set of BSs and AGs will be denoted by $\mathbb{B}$ and $\mathbb{M}$, respectively, and let $\mathbb{K}=\mathbb{B} \cup \mathbb{M}$. A node $v$ is considered as the neighbor of AG $u$ if AG $u$ can communicate and range with $v$. The neighboring node set of AG $u$ at time step $k$ is denoted as $\mathbb{K}_{u}^{(k)}$, allowing us to define $\mathbb{M}_{u}^{(k)}=\mathbb{M} \cap \mathbb{K}_{u}^{(k)}$ and $\mathbb{B}_{u}^{(k)}=\mathbb{B} \cap \mathbb{K}_{u}^{(k)}$. Finally, we denote the set of connected node pairs at time step $k$ by $\mathbb{P}^{(k)}$.

For a generic node $u$, the state space corresponds to the two-dimensional position, i.e., $\mathbf{p}_{u}^{(k)}=\left[x_{u}, y_{u}\right]^{\mathrm{T}}$. The positions of BSs are assumed to be known and fixed. The positions of agents are controlled as follows: agent $u$ moves step-wise with a control command $\mathbf{u}_{u}^{(k)}$ and Gaussian transition noise $\boldsymbol{\epsilon}_{u}^{(k)} \sim \mathcal{N}\left(\mathbf{0}, \mathbf{Q}_{u}\right)$

$$
\mathbf{p}_{u}^{(k)}=\mathbf{p}_{u}^{(k-1)}+\mathbf{u}_{u}^{(k)}+\boldsymbol{\epsilon}_{u}^{(k)}, \quad u \in \mathbb{M} .
$$

The global state $\mathbf{p}^{(k)}$ of all AGs is obtained by stacking the states of the individual agents into a vector, and can thus be expressed as

$$
\mathbf{p}^{(k)}=\mathbf{p}^{(k-1)}+\mathbf{u}^{(k)}+\boldsymbol{\epsilon}^{(k)},
$$

where $\boldsymbol{\epsilon}^{(k)} \sim \mathcal{N}(\mathbf{0}, \mathbf{Q})$ is the global transition noise with covariance $\mathbf{Q}=\operatorname{diag}\left[\ldots, \mathbf{Q}_{u}, \ldots\right]$. 


\section{B. Localization}

At each step, agents acquire radio-based observations from their neighboring nodes:

$$
z_{u, v}^{(k)}=h_{u, v}\left(\mathbf{p}_{u}^{(k)}, \mathbf{p}_{v}^{(k)}, \omega_{u, v}^{(k)}\right) \quad(u, v) \in \mathbb{P}^{(k)},
$$

where $h_{u, v}$ is an observation function, which depends on the measurement method and $\omega_{u, v}^{(k)}$ is observation noise. Without loss of generality, we assume the observations are the independent propagation time-based inter-node distance estimates with

$$
\mathbb{E}\left[\left(z_{u, v}^{(k)}-d_{u, v}^{(k)}\right)^{2}\right]=\left(\sigma_{u, v}^{(k)}\right)^{2},
$$

where $d_{u, v}$ is the distance between AG $u$ and node $v$. The ranging variance $\left(\sigma_{u, v}^{(k)}\right)^{2}$ is, in general, distance dependent by the radio propagation model [8]. The global observation $\mathbf{z}^{(k)}$ is obtained by stacking all the observations into a global vector. With an associated likelihood function the observation likelihood is written as:

$$
p\left(\mathbf{z}^{(k)} \mid \mathbf{p}^{(k)}\right)=\prod_{(u, v) \in \mathbb{P}^{(k)}} p\left(z_{u, v}^{(k)} \mid \mathbf{p}_{u}^{(k)}, \mathbf{p}_{v}^{(k)}\right) .
$$

The positions of the AGs can be estimated in either snapshot or Bayesian fashion. In this work, our focus is on the snapshot estimation case, where positions are estimated independently at each step, for example, by a maximum likelihood (ML) estimator

$$
\hat{\mathbf{p}}^{(k)}=\underset{\mathbf{p}^{(k)}}{\arg \max } p\left(\mathbf{z}^{(k)} \mid \mathbf{p}^{(k)}\right) .
$$

We assume that all nodes are perfectly synchronized.

\section{Problem Formulation}

At each time step $k$, a global control command $\mathbf{u}^{(k)}$ must be generated so that the swarm navigates itself from the initial positions $\mathbf{p}^{(0)}$ to a pre-defined destination $\mathbf{q} \in \mathbb{R}^{2}$. Meanwhile the localization error of all AGs is minimized. The goal of this paper is to design such a controller.

A swarm system with three AGs and two BSs is illustrated in Fig. 1. Three AGs move from time step $k-1$ to time step $k$ towards the BSs. Solid lines indicate the connections among nodes. dash lines with arrows show the control command for AG $1, \mathbf{u}_{1}^{(k)}$, and the distortion on that controller $\epsilon_{1}^{(k)}$.

\section{Controller Design}

\section{A. High-level Description}

The global control command $\mathbf{u}^{(k)}$ is generated based on the objectives of the application and the position estimates at previous step $\hat{\mathbf{p}}^{(k-1)}$ to decide where to go next. We consider an alternating optimization approach, where the swarm alternately solves two problems:

$$
\begin{array}{cl}
\text { Problem } \mathcal{P}_{\alpha}: & \underset{\mathbf{u}_{\alpha}^{(k)}}{\operatorname{minimize}}\left\|\mathbf{p}^{(k)}-\mathbf{1}_{M \times 1} \otimes \mathbf{q}\right\| \\
& \text { subject to } \mathbf{u}_{\alpha}^{(k)} \in \mathcal{U}_{\alpha}
\end{array}
$$

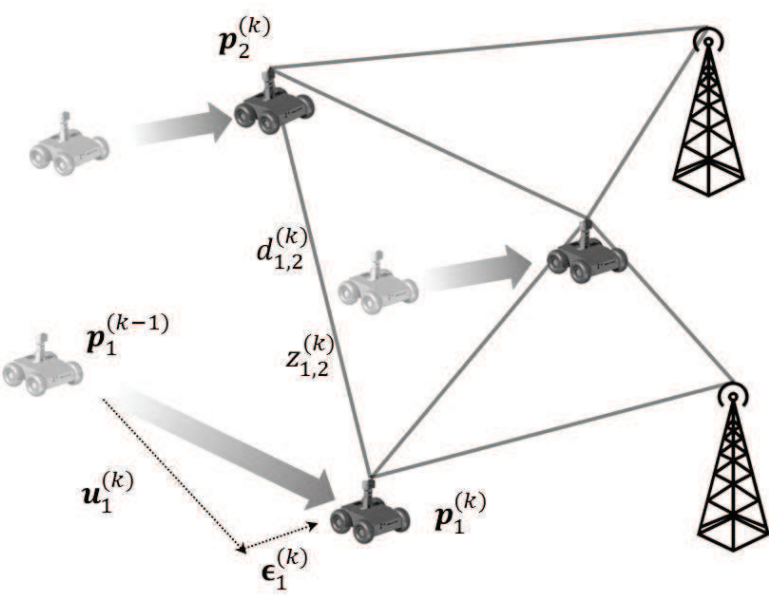

Figure 1. A swarm navigation system with three AGs and two BSs

to move all agents towards the destination $\mathbf{q}$, and

$$
\begin{array}{cl}
\text { Problem } \mathcal{P}_{\beta}: & \underset{\mathbf{u}_{\beta}^{(k)}}{\operatorname{minimize}}\left\|\hat{\mathbf{p}}^{(k)}-\mathbf{p}^{(k)}\right\| \\
& \text { subject to } \mathbf{u}_{\beta}^{(k)} \in \mathcal{U}_{\beta}
\end{array}
$$

to minimize the swarm localization error.

The symbol $\otimes$ denotes the Kronecker product. The term $\mathbf{1}_{M \times 1} \otimes \mathbf{q}$ stacks the goal position into a vector with the same size as $\mathbf{p}^{(k)}$. Note that the dependence on the optimization variable $\mathbf{u}_{\alpha}^{(k)}$ in Problem $\mathcal{P}_{\alpha}$ is implicitly given by the incorporation of (2), and similarly for $\mathcal{P}_{\beta}$. The set of admissible controls is defined as $\mathcal{U}_{\alpha}=\left\{\mathbf{u} \in \mathbb{R}^{2 M} \mid\|\mathbf{u}\|=\mu_{\alpha}\right\}$ and similarly for $\mathcal{U}_{\beta}$. Solving Problem $\mathcal{P}_{\alpha}$ and $\mathcal{P}_{\beta}$ in an alternating manner thus aims at moving the AGs towards the goal $\mathbf{q}$ and minimizing the AGs position uncertainty.

The solution of Problem $\mathcal{P}_{\alpha}$ (i.e., the control command $\left.\mathbf{u}_{\alpha}^{(k)}\right)$ is easily generated from the differences between position estimates and the destination:

$$
\mathbf{u}_{\alpha}^{(k)}=-\mu_{\alpha} \frac{\hat{\mathbf{p}}^{(k-1)}-\mathbf{1}_{M \times 1} \otimes \mathbf{q}}{\left\|\hat{\mathbf{p}}^{(k-1)}-\mathbf{1}_{M \times 1} \otimes \mathbf{q}\right\|},
$$

where $\mu_{\alpha}$ is the step size. Therefore, in the next section, we focus on solving Problem $\mathcal{P}_{\beta}$.

\section{B. Localization Driven Formation Optimization}

To solve $\mathcal{P}_{\beta}$, we will rely on the theory of Fisher information and the CRB. The CRB is a lower bound on the estimation error variance of any unbiased estimator, and is expressed as

$$
\mathbb{E}\left[\left\|\hat{\mathbf{p}}^{(k)}-\mathbf{p}^{(k)}\right\|^{2}\right] \geq \operatorname{CRB}\left[\mathbf{p}^{(k)}\right]=\operatorname{tr}\left(\left(\mathbf{F}_{\mathbf{p}}^{(k)}\right)^{-1}\right),
$$

where $\mathbf{F}_{\mathbf{p}}^{(k)}$ is the position Fisher information matrix (FIM), defined as

$$
\mathbf{F}_{\mathbf{p}}^{(k)}=\mathbf{H}^{(k)} \underbrace{\mathbb{E}\left[-\Delta_{\mathbf{d}^{(k)}}^{\mathbf{d}^{(k)}} \ln p\left(\mathbf{z}^{(k)} \mid \mathbf{d}^{(k)}\right)\right]}_{=\mathbf{F}_{\mathbf{d}}^{(k)}}\left(\mathbf{H}^{(k)}\right)^{\mathrm{T}},
$$

in which $\mathbf{d}^{(k)}$ is the vector of true distances for links in $\mathbb{P}^{(k)}$, $\nabla_{\mathbf{a}}$ is the gradient operation with respect to $\mathbf{a}$ and $\Delta_{\mathbf{a}}^{\mathbf{b}} \triangleq$ 
$\nabla_{\mathbf{a}} \nabla_{\mathbf{b}}^{T}$ is the Hessian matrix with respect to $\mathbf{a}$ and $\mathbf{b}$, and $\mathbf{H}^{(k)}$ is the geometry matrix, expressing the effect from the geometry relation among the nodes:

$$
\mathbf{H}^{(k)}=\nabla_{\mathbf{p}^{(k)}}\left(\mathbf{d}^{(k)}\right)^{\mathrm{T}} .
$$

Given the model from Section II-B, $\mathbf{F}_{\mathbf{d}}^{(k)}$ is the diagonal ranging FIM, with the entries along the diagonal being the inverse of the ranging CRB for every link. $\mathbf{F}_{\mathbf{d}}^{(k)}$ shows the impact from the distance estimate accuracy. For simplicity, we will omit the superscript $(k)$ if there is no ambiguity.

We can reformulate $\mathcal{P}_{\beta}$ with the position FIM as

$$
\underset{\mathbf{u}_{\beta} \in \mathcal{U}_{\beta}}{\operatorname{minimize}} \operatorname{tr}\left(\mathbf{F}_{\mathbf{p}}^{-1}\right) .
$$

Note that, the ranging model is in general non-Gaussian. However, it has been proved in [14] that, with a fixed variance, the CRB gets its largest value under the Gaussian assumption. This allows us to replace $\mathbf{F}_{\mathbf{d}}$ with the diagonal FIM $\tilde{\mathbf{F}}_{\mathbf{d}}$ of a distance-dependent Gaussian ranging model to simplify the problem in (13). Under such a conservative Gaussian assumption, the $l$-th diagonal entry in $\tilde{\mathbf{F}}_{\mathbf{d}}$, which corresponds to link $(u, v)$, can be derived as in [15, eq. (3.31)]

$$
\lambda_{u, v}^{-2} \triangleq\left(\tilde{\mathbf{F}}_{\mathbf{d}}\right)_{l, l}=\sigma_{u, v}^{-2}+\frac{1}{2}\left(\frac{\partial \sigma_{u, v}^{2}}{\partial d_{u, v}}\right)^{2} / \sigma_{u, v}^{4} .
$$

The modified problem becomes

$$
\underset{\mathbf{u}_{\beta} \in \mathcal{U}_{\beta}}{\operatorname{minimize}} \operatorname{tr}\left(\tilde{\mathbf{F}}_{\mathbf{p}}^{-1}\right),
$$

where

$$
\tilde{\mathbf{F}}_{\mathbf{p}}=\mathbf{H} \tilde{\mathbf{F}}_{\mathbf{d}} \mathbf{H}^{\mathrm{T}} .
$$

The solution of (15) is also valid for the problem in (13) because it can be considered as the worst case and the solution will be the min-max solution for (13). We will now proceed to solve the modified problem in (15), which is a highly nonconvex problem. We propose a gradient approach to find the locally optimal solution similar to the scheme in [5]. In [5], $\tilde{\mathbf{F}}_{\mathbf{d}}$ is assumed to be a scaled identity matrix, i.e., $\tilde{\mathbf{F}}_{\mathbf{d}}=\sigma^{-2} \mathbf{I}$, indicating every link has the same distance-independent ranging variance $\sigma^{2}$. Here, we extend the formation optimization approach in [5] with a more realistic radio ranging model, where $\tilde{\mathbf{F}}_{\mathbf{d}}$ is a diagonal matrix with entries given by (14).

The gradient $\mathbf{c} \in \mathbb{R}^{2 M}$ of the objective function is

$$
\mathbf{c}=\left[\mathbf{c}_{1}^{\mathrm{T}}, \ldots, \mathbf{c}_{u}^{\mathrm{T}}, \ldots, \mathbf{c}_{M}^{\mathrm{T}}\right]^{\mathrm{T}}=\nabla_{\mathbf{p}} \operatorname{tr}\left(\tilde{\mathbf{F}}_{\mathbf{p}}^{-1}\right),
$$

where $\mathbf{c}_{u} \in \mathbb{R}^{2}$ is the gradient component of AG $u$, defined in Theorem III.1. The steepest descent gradient controller solving $\mathcal{P}_{\beta}$ with a step size $\mu_{\beta}$ can be expressed as

$$
\mathbf{u}_{\beta}=-\mu_{\beta} \frac{\mathbf{c}}{\|\mathbf{c}\|} .
$$

Theorem III.1 (Gradient of CRB). When $\tilde{\mathbf{F}}_{\mathbf{d}}$ is a diagonal matrix with entries $\lambda_{u, v}^{-2}$, in which $\lambda_{u, v}^{-2}$ may be a function of the distance $d_{u, v}$, then the $u$-th entry $\mathbf{c}_{u} \in \mathbb{R}^{2}$ of the gradient $\mathbf{c}=\nabla_{\mathbf{p}} \operatorname{tr}\left(\tilde{\mathbf{F}}_{\mathbf{p}}^{-1}\right)$ is given by

$$
\begin{aligned}
\mathbf{c}_{u}= & -\sum_{v \in \mathbb{K}_{u}} 2 \frac{\left(\mathbf{I}-\mathbf{e}_{u, v} \mathbf{e}_{u, v}^{\mathrm{T}}\right) \mathbf{Y}_{u, v} \mathbf{e}_{u, v}}{\lambda_{u, v}^{2} d_{u, v}} \\
& +\frac{\partial \lambda_{u, v}^{-2}}{\partial d_{u, v}} \mathbf{e}_{u, v} \mathbf{e}_{u, v}^{\mathrm{T}} \mathbf{Y}_{u, v} \mathbf{e}_{u, v},
\end{aligned}
$$

where $\mathbf{e}_{u, v}=\left(\mathbf{p}_{u}-\mathbf{p}_{v}\right) /\left\|\mathbf{p}_{u}-\mathbf{p}_{v}\right\|$ and $\mathbf{Y}_{u, v}$ is defined in (26)-(27).

Proof. See Appendix A.

Since the true positions of the AGs are unknown, the gradient $\mathbf{c}$ from (19) is evaluated in the position estimates $\hat{\mathbf{p}}$ and then utilized to generate the control command $\mathbf{u}_{\beta}$ through (18).

\section{Numerical Results}

\section{A. Setup and Evaluation Metrics}

Ranging Model: We conduct numerical simulations to assess the performance of the proposed formation controller. The distance estimate is acquired from a radio-based ranging signal. The signal is OFDM modulated, attenuated with a free-space pathloss model, and distorted with additive white Gaussian noise (AWGN). At a reference distance $d_{0}=20 \mathrm{~m}$, the ranging variance is $\sigma_{0}^{2}=0.01 \mathrm{~m}^{2}$. In order to analytically calculate (19), the distance dependent ranging variance of a generic link $(u, v)$ is approximated as

$$
\sigma_{u, v}^{-2}=\frac{d_{0}^{2}}{\sigma_{0}^{2} d_{u, v}^{2}} \exp \left(-\left(\frac{\sigma_{0}^{2} d_{u, v}^{2}}{d_{0}^{2}}+\left(1-\sigma_{\mathrm{di}}^{2}\right)\right)^{\gamma}\right)+\frac{1}{\sigma_{\infty}^{2}}
$$

in which $\sigma_{\mathrm{di}}^{2}=0.16 \mathrm{~m}^{2}$ is the ranging variance where distance estimate starts diverging from the ranging CRB, $\gamma=30$ controls the slope of the divergence, and $\sigma_{\infty}^{2}=1.225 \times 10^{7} \mathrm{~m}^{2}$ is the maximum ranging variance defined by the a-priori knowledge of the true distance [13]. The analytical model proposed in (20) is designed to fit the performance of a correlation-based estimator, as in [8]. The analytical model, the estimation result and the ranging CRB are shown in Fig. 2. The model captures the main features of radio-based ranging: For short distances, the ranging variance is quadratically proportional to the distance. After a certain distance, the ranging variance rapidly increases to the maximum ranging variance due to the low signal-to-noise ratio (SNR). In this paper, we use the model in (20) to emulate the ranging performance in all SNR regions. We found that under the model (20), the first term in (14) dominates the second term, and approximate $\lambda_{u, v}^{-2}$ by $\sigma_{u, v}^{-2}$.

Simulation Scenario: The simulation scenario is illustrated in Fig. 3, where 25 AGs, shown as red dots, need to move from region $A$ to region $B$ based on their position estimates. As initialization, AGs are uniformly deployed in an area of $10 \mathrm{~m} \times 10 \mathrm{~m}$ at region $A$. 


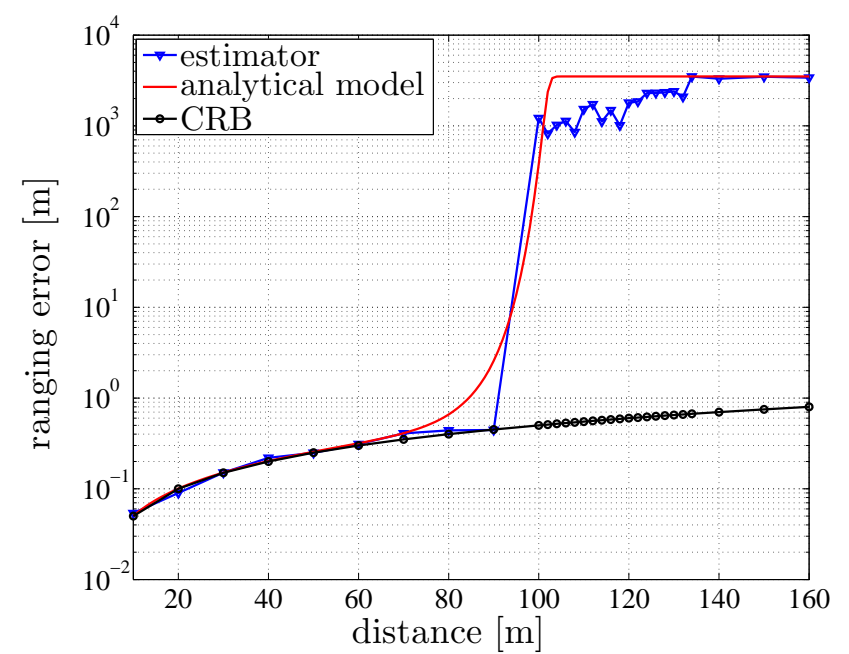

Figure 2. Modeling of the ranging error variance with distance: the CRB leads to overly optimistic errors at large distances. The proposed analytical model (20) can capture the behavior of practical estimators at both large and small distances.

In each region three $\mathrm{BSs}$, shown as red triangles, are deployed. The gray shaded background is the value of the non-cooperative position CRB (i.e., obtained by performing measurements only with the anchors). There is a blind region between $x=100 \mathrm{~m}$ and $x=200 \mathrm{~m}$, in which none of the AGs can effectively connect to any BS due to low SNR. The step sizes are set to $\mu_{\alpha}=6.25 \mathrm{~m}$ and $\mu_{\beta}=12.5 \mathrm{~m}$. Hence at each step, an AG moves, on average, $0.25 \mathrm{~m}$ for the $\alpha$ objective (solving $\mathcal{P}_{\alpha}$ ) and then $0.5 \mathrm{~m}$ for the $\beta$ objective (solving $\mathcal{P}_{\beta}$ ), if applied. Transition noise variance for a single AG at each dimension is set to $0.01 \mathrm{~m}^{2}$, i.e., $\quad \mathbf{Q}_{u}=\operatorname{diag}[0.01,0.01]$. Three control strategies are compared, namely (i) only $\alpha$ objectives, (ii) alternating $\alpha$ and $\beta$ objectives, assuming ranging FIM is a scaled identity matrix, i.e. approach in [5], and (iii) our proposed alternating $\alpha$ and $\beta$ objectives scheme with the realistic ranging assumption, i.e. based on (14), (19) and (20). Simulations are repeated 100 times to obtain statistical results.

Performance Metrics: We introduce two metrics to evaluate the performance, namely the remaining distance and the expected position root mean-square error (RMSE). The remaining distance is defined as the difference between an AG's position and the destination, showing the accomplishment of the $\alpha$ objective. The expected position RMSE is calculated from the square root of the trace of positioning $\mathrm{CRB}$, indicating the $\beta$ objective performance.

\section{B. Discussion}

We first evaluate the snapshot formations for one realization at time step $k=150$. Fig. 3(a) shows the result when AGs move with strategy (i), while Fig. 3(b) shows the result when AGs move with strategy (iii). Green circles show the expected position estimates generated from the cooperative positioning CRB. When only the $\alpha$ objective is used, all AGs are in a blind region (Fig. 3(a)), so that no effective reception from any

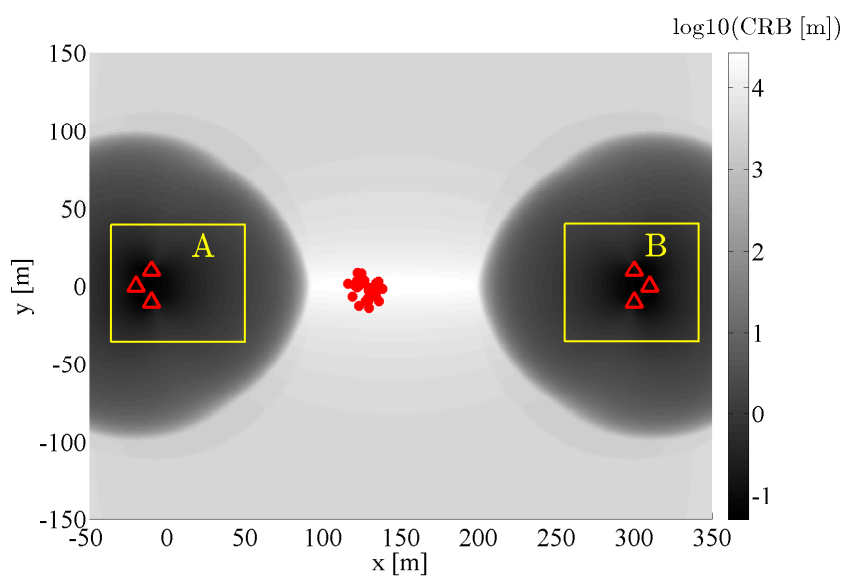

(a) strategy (i)

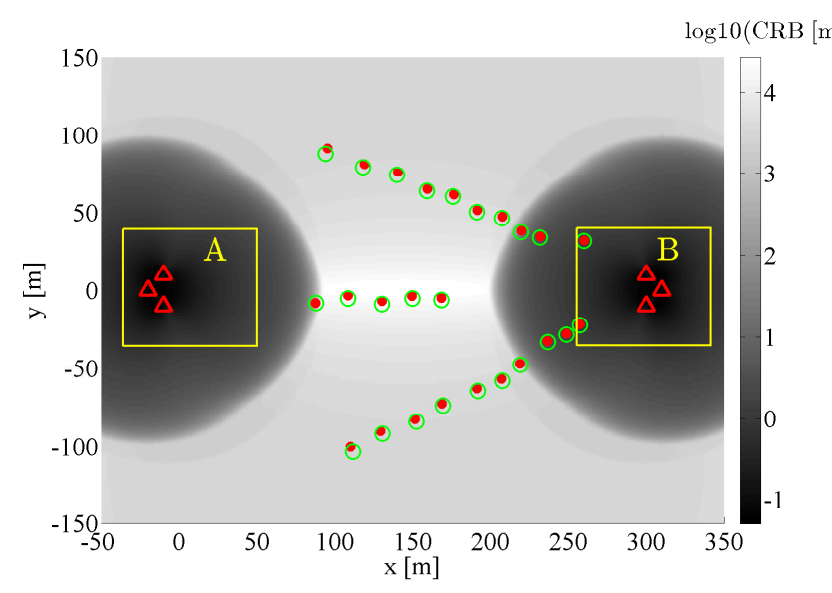

(b) strategy (iii)

Figure 3. Formation at step $k=150$, red triangles are the positions of BSs, red dots are the positions of AGs, green circles show the expected formation estimates generated from the cooperative positioning CRB. The gray shaded background is the value of the non-cooperative position CRB (i.e., obtained by performing measurements only with the anchors).

of the BSs is possible. Therefore, the position estimates are heavily biased, too far to be illustrated in the plot. Based on the biased position estimates, an effective control command cannot be generated. Hence, the $\alpha$ objective is not accomplished. When the proposed strategy (iii) is applied (Fig. 3(b)), the swarm automatically emerges to some chains along the $x$ dimension, to propagate high localization accuracy into the blind region. The chains are distanced, but yet connected, in the $y$ dimension to gain a good geometric dilution of precision (GDOP). With this formation, the swarm collaboratively forms a virtual bridge through the blind region in order to reach the goal, while maintaining precise localization.

In Fig. 4, the overall mean (lines), maximal (upper end of the bars) and minimal (lower end of the bars) remaining distances to the goal position for all three strategies are plotted. When strategies (i) and (ii) are employed, the remaining 


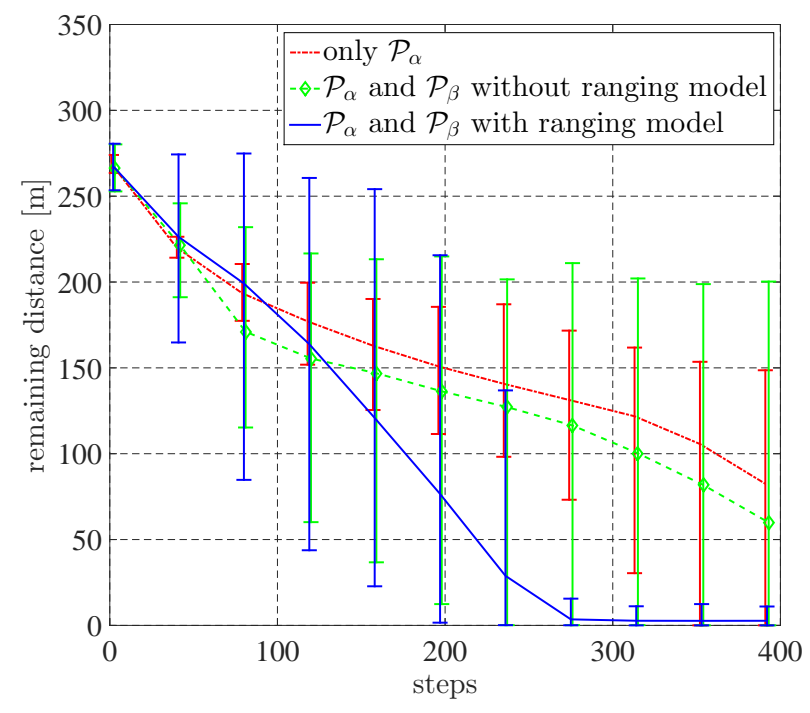

Figure 4. Mean (lines), maximal (upper end of the bars) and minimal (lower end of the bars) remaining distance to the goal comparison for all three strategies

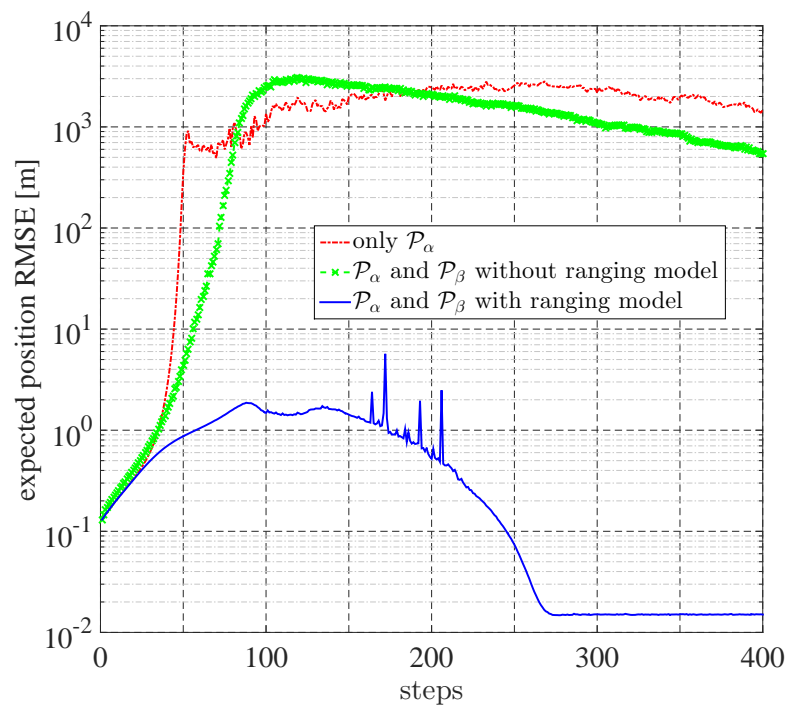

Figure 5. Expected position RMSE comparison for all three strategies, generated from the global position CRB.

distances after 400 time steps are large (up to $150 \mathrm{~m}$ from the destination). At the beginning (the first 100 steps), the AGs move towards the destination. When they all step in the blind region, the remaining distances start diverging due to the localization imprecision. When the proposed strategy (iii) is applied, the AGs first spread out and then all reach the destination. While moving towards the destination, AGs span into a larger area to exploit the spatial diversity. Some AGs arrive at the destination within 200 steps, with precise localization supported by the AGs left behind. Then the AGs, who have arrived at the goal, support the others to pass the blind region. After less than 300 steps, the remaining distances converge to a small value, hence all AGs reach the goal position.

The expected position RMSE comparison for all three strategies is shown in Fig. 5. For the (i) and (ii) strategies, the position RMSE quickly rises to above $1000 \mathrm{~m}$ due to the loss of BS reception and remains high for the rest of the steps. Strategy (ii) slightly outperforms (i). However, due to a overlooking of the ranging model, strategy (ii) fails to generate effective control as well. For the proposed strategy (iii), the position RMSE slowly increases to around two meters. After 100 steps, the curve slightly drops to $1.5 \mathrm{~m}$ and rises back to two meters again. It shows a handover process, in which the swarm switches the BSs connection from the area $A$ to the area $B$. During this process, the swarm maintains effective connection to the BSs in both area. After 150 steps, the curve drops down with some spikes up to five meters. The spikes do not show a significant impact to the performance and can even be eliminated with a Bayesian estimator in a real application. The position RMSE curve is stabilized to a centimeter level after less than 300 steps, which coincides with the remaining distance curve in Fig. 4, indicating the swarm successfully reaches the goal position with precise localization.

\section{CONCLUSION}

We have presented a framework that combines goalapproaching with localization-driven formation control for a swarm migration application, with a distance-dependent radiobased ranging model. The resulting controller accounts for position uncertainty and, while agents move towards goals, emerges formations that lead to high-precision localization performance. From the numerical results we can conclude that, a realistic ranging model is essential for generating an effective controller. Overlooking either geometry or radio ranging characteristic leads to a failure of swam navigation. The swarm migration is accomplished by controlling the formation with the goal-approaching and the localization-orientated objectives together, under a realistic ranging model assumption.

\section{APPENDIX A \\ PROOF OF THEOREM III.1}

We define $s=\left(\mathbf{p}_{u}\right)_{d}$ as the $d$-th dimension of AG $u$ 's state. The derivative of the objective function in (15) with respect to $s$ is stated as

$$
\frac{\partial \operatorname{tr}\left(\tilde{\mathbf{F}}_{\mathbf{p}}^{-1}\right)}{\partial s}=-\operatorname{tr}(\underbrace{\tilde{\mathbf{F}}_{\mathbf{p}}^{-1} \tilde{\mathbf{F}}_{\mathbf{p}}^{-1}}_{\triangleq \mathbf{X}} \frac{\partial \tilde{\mathbf{F}}_{\mathbf{p}}}{\partial s}) .
$$

We further define $\mathbf{X}_{m, n} \in \mathbb{R}^{2 \times 2}$ as the sub-matrix $\mathbf{X}_{2 m-1: 2 m, 2 n-1: 2 n}$, and $\mathbf{F}_{m, n} \in \mathbb{R}^{2 \times 2}$ as the sub-matrix $\left(\tilde{\mathbf{F}}_{\mathbf{p}}\right)_{2 m-1: 2 m, 2 n-1: 2 n}$, expressed as

$$
\begin{aligned}
\mathbf{F}_{m, m} & =\sum_{n \in \mathbb{K}_{m}} \frac{1}{\lambda_{m, n}^{2}} \mathbf{e}_{m, n} \mathbf{e}_{m, n}^{\mathrm{T}}, \\
\mathbf{F}_{m, n} & =-\frac{1}{\lambda_{m, n}^{2}} \mathbf{e}_{m, n} \mathbf{e}_{m, n}^{\mathrm{T}}, \quad n \in \mathbb{K}_{m} .
\end{aligned}
$$


Noticing the following properties $\mathbf{X}_{m, n}=\mathbf{X}_{n, m}^{\mathrm{T}}$ and $\mathbf{F}_{m, n}=$ $\mathbf{F}_{m, n}^{\mathrm{T}}=\mathbf{F}_{n, m}$, (21) can be rewritten as

$$
\begin{aligned}
\frac{\partial \operatorname{tr}\left(\tilde{\mathbf{F}}_{\mathbf{p}}^{-1}\right)}{\partial s} & =-\operatorname{tr} \sum_{(m, n) \in \mathbb{P}} \mathbf{X}_{m, n} \frac{\partial \mathbf{F}_{m, n}}{\partial s} \\
& =-\operatorname{tr} \sum_{v \in \mathbb{K}_{u}} \mathbf{Y}_{u, v} \frac{\partial \lambda_{u, v}^{-2} \mathbf{e}_{u, v} \mathbf{e}_{u, v}^{\mathrm{T}}}{\partial s}
\end{aligned}
$$

where we have introduced

$$
\begin{aligned}
\mathbf{Y}_{u, v} & =\mathbf{X}_{u, u}-\mathbf{X}_{u, v}-\mathbf{X}_{v, u} & & v \in \mathbb{M}_{u} \\
\mathbf{Y}_{u, v} & =\mathbf{X}_{u, u} & & v \in \mathbb{B}_{u} .
\end{aligned}
$$

Considering the dependence of $\lambda_{u, v}^{2}$ on $d_{u, v}$, (24) becomes

$$
\begin{aligned}
& \frac{\partial \operatorname{tr}\left(\tilde{\mathbf{F}}_{\mathbf{p}}^{-1}\right)}{\partial s} \\
= & -\sum_{v \in \mathbb{K}_{u}} 2 \frac{1}{\lambda_{u, v}^{2}} \frac{\partial \mathbf{e}_{u, v}^{\mathrm{T}}}{\partial s} \mathbf{Y}_{u, v} \mathbf{e}_{u, v}+\frac{\partial \lambda_{u, v}^{-2}}{\partial s} \mathbf{e}_{u, v}^{\mathrm{T}} \mathbf{Y}_{u, v} \mathbf{e}_{u, v}
\end{aligned}
$$

Finally, we can express the gradient of $\tilde{\mathbf{F}}_{\mathbf{p}}^{-1}$ with respect to the state of AG $u$ by (19), which completes the proof.

\section{ACKNOWLEDGMENT}

This work was partially supported by the European Commission, under EU FP7 Marie Curie Initial Training Network MULTI-POS (Multi-technology Positioning Professionals) under grant nr. 316528, the European Research Council, under grant nr. 258418 (COOPNET), the EU project HIGHTS (High precision positioning for cooperative ITS applications) MG3.5a-2014-636537 and the DLR project Dependable Navigation.

\section{REFERENCES}

[1] J. S. Shamma, Cooperative control of distributed multi-agent systems. Wiley Online Library, 2007.

[2] R. Olfati-Saber, "Flocking for Multi-Agent Dynamic Systems: Algorithms and Theory," Automatic Control, IEEE Transactions on, vol. 51, no. 3, pp. 401-420, 2006.

[3] N. M. M. de Abreu, "Old and new results on algebraic connectivity of graphs," Linear algebra and its applications, vol. 423, no. 1, pp. 53-73, 2007.

[4] Y. Kim and M. Mesbahi, "On maximizing the second smallest eigenvalue of a state-dependent graph Laplacian," IEEE Transactions on Automatic Control, vol. 51, no. 1, pp. 116-120, 2006.

[5] Y. Kim, G. Zhu, and J. Hu, "Optimizing formation rigidity under connectivity constraints," in IEEE Conference on Decision and Control (CDC), 2010, pp. 6590-6595.

[6] M. Zavlanos, M. Egerstedt, and G. Pappas, "Graph-theoretic connectivity control of mobile robot networks," Proceedings of the IEEE, vol. 99, no. 9, pp. 1525-1540, 2011.

[7] H. Wymeersch, J. Lien, and M. Win, "Cooperative Localization in Wireless Networks," Proceedings of the IEEE, vol. 97, no. 2, pp. 427 -450 , Feb. 2009.

[8] S. Zhang, R. Raulefs, A. Dammann, and S. Sand, "System-Level Performance Analysis for Bayesian Cooperative Positioning: From Global to Local," in Proceeedings of 2013 International Conference on Indoor Position and Indoor Navigation (IPIN), 2013.

[9] Y. Shen, H. Wymeersch, and M. Win, "Fundamental limits of wideband localization - part ii: Cooperative networks," Information Theory, IEEE Transactions on, vol. 56, no. 10, pp. 4981 -5000, oct. 2010.
[10] S. Zhang and R. Raulefs, "Multi-agent flocking with noisy anchor-free localization," in 11th International Symposium on Wireless Communications Systems (ISWCS), 2014, pp. 927-933.

[11] F. Morbidi and G. L. Mariottini, "Active target tracking and cooperative localization for teams of aerial vehicles," IEEE Trans. Control Syst. Technol., vol. 21, no. 5, pp. 1694-1707, 2013.

[12] F. Meyer, H. Wymeersch, M. Frohle, and F. Hlawatsch, "Distributed estimation with information-seeking control in agent networks," Selected Areas in Communications, IEEE Journal on (accepted), 2015.

[13] D. Dardari, A. Conti, U. Ferner, A. Giorgetti, and M. Win, "Ranging with Ultrawide Bandwidth Signals in Multipath Environments," Proceedings of the IEEE, vol. 97, no. 2, pp. 404-426, 2009.

[14] P. Stoica and P. Babu, "The Gaussian data assumption leads to the largest Cramér-Rao bound [lecture notes],' Signal Processing Magazine, IEEE, vol. 28, no. 3, pp. 132-133, May 2011.

[15] S. M. Kay, Fundamentals of Statistical Signal Processing: Estimation Theory. Upper Saddle River, NJ, USA: Prentice-Hall, Inc., 1993. 